\title{
Análise preliminar dos impactos do uso de mais de um sistema GNSS no posicionamento preciso
}

\author{
Samara Chamorro Navarro Ferreira* \\ Luiz Paulo Souto Fortes**
}

Recibido el 3 de junio de 2016; aceptado el 18 de septiembre de 2016

\section{Resumen}

Para la obtención de coordenadas precisas en Geodesia Satelital se utilizan las técnicas de posicionamiento relativo y de Posicionamiento Preciso por Punto (PPP). Se presenta en este trabajo un análisis preliminar de los impactos en la Dilución de la Precisión (DOP) y en la calidad del posicionamiento causados por el rastreo de diversos Sistema

Globales de Navegación por Satélites (GNSS), con la utilización de receptores de simple y doble frecuencia, considerando sesiones de dos y 24 horas de duración en dos épocas del año y, por lo tanto, sometidas a distintas condiciones ionosféricas. Estos análisis fueron realizados con base en los datos disponibles de las estaciones de la Red Brasileña de Monitoreo Continuo de los Sistemas GNSS (RBMC) y del Multi-GNSS Experiment (MGEX) del Servicio Internacional GNSS (IGS). Los resultados de los procesamientos fueron alcanzados a través de los programas/servicios GNSS Planning Online, IBGE-PPP y RTKLIB, que confirmaron la mejora de calidad de los posicionamientos relativos y PPP, cuando se cuenta con más observaciones GNSS disponibles. Otro factor que contribuye para esta mejora es la geometría más favorable de la constelación de satélites utilizada en las soluciones. La actividad solar, época del año y horario de rastreo también influyen directamente sobre la calidad del posicionamiento.

Palabras clave: GNSS, Geometría de los satélites, Posicionamiento relativo, Posicionamiento Preciso por Punto (PPP).

* Eng. Cart. Departamento de Engenharia Cartográfica, Universidade do Estado do Rio de Janeiro (UERJ), Rua São Francisco Xavier 524, Maracanã, Rio de Janeiro, RJ, CEP 20550-900, Brasil, e-mail: samarachamorro@gmail.com

** Prof., PhD, Departamento de Engenharia Cartográfica, UERJ, Brasil, e-mail: luiz.paulo.fortes@gmail.com 


\section{Abstract}

To obtain precise coordinates in Satellite Geodesy, relative positioning and precise point positioning (PPP) techniques are used. In this work a preliminary analysis of the impacts on Dilution of Precision (DOP) and positioning accuracy caused by tracking several GNSS, using single and double frequency receivers, considering two- and 24-hour sessions in two time periods of the year, and, as such, subject to different ionospheric conditions, is presented. These analyses have been performed based on data available from the Brazilian Network for Continuous Monitoring of GNSS (RBMC) and the Multi-GNSS Experiment (MGEX) of the International GNSS Service (IGS). The processing results have been obtained using the GNSS Planning Online, IBGE-PPP and RTKLIB software/services, which have confirmed the accuracy improvement in relative and PPP positioning when using more GNSS observations. Another factor which contributes to this improvement is a more favourable satellite constellation used in the solutions. The solar activity, time of year and tracking period of the day also directly impact the quality of positioning.

Key words: GNSS, Satellite Geometry, Relative Positioning, Precise Point Positioning (PPP).

\section{Resumo}

Para a obtenção de coordenadas precisas em Geodésia por Satélites utilizam-se as técnicas de posicionamento relativo e de Posicionamento Preciso por Ponto (PPP). Neste trabalho é apresentada uma análise preliminar dos impactos na Diluição da Precisão (DOP) e na qualidade do posicionamento causados pelo rastreio de diversos Sistemas Globais de Navegação por Satélites (GNSS), com uso de receptores de simples e dupla frequência, considerando sessões de duas e 24 horas de duração em duas épocas do ano e, portanto, sujeitas a diferentes condições ionosféricas. Estas análises foram realizadas com base nos dados disponíveis das estações da Rede Brasileira de Monitoramento Contínuo dos Sistemas GNSS (RBMC) e do Multi-GNSS Experiment (MGEX) do Serviço Internacional GNSS (IGS). Os resultados dos processamentos foram obtidos com o uso dos programas/serviços GNSS Planning Online, IBGE-PPP e RTKLIB, que confirmaram a melhoria da qualidade dos posicionamentos relativos e PPP quando se conta com mais observações GNSS disponíveis. Outro fator que contribui para esta melhoria é a geometria mais favorável da constelação de satélites utilizada nas soluções. A atividade solar, época do ano e horário de rastreio também influenciam diretamente a qualidade do posicionamento.

Palavras-chave: GNSS, Geometria dos satélites, Posicionamento relativo, Posicionamento Preciso por Ponto (PPP). 


\section{Introdução}

Os primeiros sistemas globais de posicionamento por satélite (GNSS, na sigla em inglês) surgiram como sistemas militares concorrentes, sendo concebidos na Guerra fria. O GPS (Global Positioning System) foi desenvolvido pelo Departamento de Defesa dos Estados Unidos da América enquanto que o GLONASS (Global Navigation Satellite System) pela extinta União Soviética. Ambos possuem características semelhantes, o que possibilita a utilização para os mesmos tipos de aplicação. Atualmente, novos sistemas globais de posicionamento por satélites estão sendo desenvolvidos, tais como o Galileo, pela União Europeia, e o BeiDou, pela China, porém apenas o GPS e o GLONASS encontram-se plenamente operacionais.

Espera-se que a disponibilidade de satélites proporcionada pelo uso combinado dos diversos sistemas GNSS ofereça uma melhoria da geometria da constelação utilizada na solução, especialmente em áreas com visibilidade restrita, maior rapidez, qualidade e robustez no posicionamento, etc. $\mathrm{O}$ uso integrado de mais de um sistema requer a consideração de vários aspectos, tais como compatibilização dos sistemas de referência, características de transmissão dos sinais, programas para processamento de dados, entre outras características. Desta forma, usá-los em conjunto constitui um desafio, mas traz avanços para a comunidade usuária.

Nesse contexto, este trabalho objetiva descrever brevemente os sistemas operacionais e em desenvolvimento que fazem parte do GNSS e analisar a qualidade e a geometria ao se usar mais de um sistema nas atividades de posicionamento. Os sistemas abordados são GPS, GLONASS, Galileo e BeiDou.

A partir dos resultados do processamento dos dados destes sistemas GNSS, buscou-se chegar a indicações a respeito da qualidade do posicionamento considerando-se o uso do GPS isoladamente e em conjunto com os demais.

\section{Introdução ao GNSS}

Em 1973, surgiu a proposta do GPS, um sistema de radionavegação desenvolvido pelo Departamento de Defesa dos Estados Unidos, com intuito de ser o principal sistema de navegação das forças armadas norte americanas (Parkinson, 1996). O sinal transmitido por ele apresentava alta qualidade, e por isso foi o precursor na evolução do posicionamento geodésico e de outros sistemas de navegação.

Inicialmente para fins militares, assim como o GPS, surgiu o GLONASS, desenvolvido pela extinta União Soviética. O primeiro satélite foi lançado em 1982. O número de satélites foi gradualmente aumentando até obter-se uma constelação entre 10 a 12 satélites, que permitiu definir o sistema como operacional (mas não com cobertura global). Após alcançar uma constelação completa com 24 satélites em 1995, a crise econômica da Rússia provocou a redução gradual do número de 
satélites. A partir de 2003 uma nova geração de satélites foi lançada e em 2011 o sistema tornou-se completamente operacional de novo.

O termo GNSS (Global Navigation Satellite Systems) foi utilizado em 1991 pela Associação Internacional de Aviação Civil para designar os sistemas de posicionamento por satélites artificiais com cobertura mundial. Os sistemas GPS e GLONASS foram os pioneiros nesse conceito e encorajaram o surgimento de outros sistemas, tais como o Galileo (União Europeia) e BeiDou (China).

\section{GPS}

O GPS tornou-se totalmente operacional in 1995 com 24 satélites localizados à altitude aproximada de $20.200 \mathrm{~km}$, distribuídos por seis planos orbitais espaçados em $60^{\circ}$ entre si e quatro satélites por plano, com inclinação de $55^{\circ}$ em relação ao plano equatorial, e com período orbital de aproximadamente 12 horas siderais. Dessa forma, a distribuição dos satélites permite que em qualquer ponto do globo, a qualquer momento, estejam disponíveis no mínimo quatro satélites GPS. Cada satélite GPS transmite duas ondas portadoras L1 e L2, com frequência de 1575,42 $\mathrm{MHz}$ e 1227,6 MHz, e comprimento de onda de $19 \mathrm{~cm}$ e $24 \mathrm{~cm}$, respectivamente. Essas frequências permitem que o usuário corrija grande parte dos erros provocados pela refração ionosférica. Os militares autorizados utilizavam o código $\mathrm{P}$ em ambas as frequências, os civis tinham acesso apenas ao sinal L1 e do código C/A. Com a modernização do GPS, foi incluído o novo código L2C para os civis na portadora L2 e um novo sinal L5, espera-se melhorias na qualidade do posicionamento para os usuários civis (Monico, 2008).

\section{GLONASS}

A constelação nominal do GLONASS é constituída por 24 satélites, sendo três de reserva $(21+3)$. Os satélites estão dispostos em três planos orbitais espaçados $120^{\circ}$ entre si. Os 21 satélites asseguram, em 97\% dos casos, que pelo menos quatro satélites estejam visíveis em qualquer ponto da superfície da Terra. Assim como ocorre com o GPS, os satélites encontram-se em órbita MEO (Medium Earth Orbit), a cerca de $19.100 \mathrm{~km}$ da superfície da Terra, com inclinação de 64,8 . O período orbital do GLONASS é de 11 horas e 15 minutos (Figueirêdo, 2005). O sistema GLONASS transmite sinais em duas frequências, L1 e L2, com 1602 Mhz e $1246 \mathrm{Mhz}$, respectivamente (Gualdino, 2011).

\section{Galileo}

O programa europeu Galileo é responsável pelo desenvolvimento do primeiro sistema para atender primordialmente as necessidades do usuário civil. Quando estiver totalmente operacional, o sistema será composto por uma constelação de 30 
satélites em órbita $\mathrm{MEO}$, em três planos inclinados $56^{\circ}$ em relação ao plano equatorial, a $23.222 \mathrm{~km}$ de altitude. Cada plano conterá 10 satélites, sendo um deles de reserva. Este último satélite terá a capacidade de substituir qualquer outro satélite do mesmo plano. Atualmente, existem 14 satélites do programa Galileo em órbita. O Galileo fornecerá seis sinais de navegação nas faixas de frequências 1164 - $1215 \mathrm{MHz}$ (banda E5), 1260 - $1300 \mathrm{MHz}$ (banda E6), e 1559 - $1592 \mathrm{MHz}$ (banda E2-L1-E1 - ou simplesmente L1). De forma a minimizar a complexidade e custo de implementação dos receptores, os sinais Galileo E5a e E2-L1-E1 serão transmitidos usando frequências idênticas às dos sinais GPS L5 e L1, respectivamente.

\section{BeiDou/Compass}

O sistema de posicionamento BeiDou foi proposto pela academia chinesa Chen Fangyun em 1983, com o intuito de oferecer suporte à navegação de navios da marinha chinesa. O BeiDou encontra-se em desenvolvimento, possuindo atualmente 18 satélites operacionais MEO, geoestacionários e geossíncronos. O BeiDou opera nas faixas de frequência do GPS L1 e L2 e é usado para melhorar a precisão de dados obtidos através dos sistemas GPS e GLONASS. O sistema é caracterizado por uma dupla utilização (militar e civil). O serviço civil foi declarado operacional (a nível regional) após o lançamento do terceiro satélite em Junho de 2003. Em 2007 iniciou-se o programa Compass, que corresponde à segunda geração do BeiDou, com o envio do primeiro satélite em órbita MEO, a $21.500 \mathrm{Km}$ de altitude. As bandas de frequência do sistema Compass sofreram alterações em relação à primeira geração do sistema BeiDou, passando a utilizar três bandas (1559.05 1591.79 MHz; 1166.22-1217.37 MHZ; 1250.62-1286.42 MHz), sendo a terceira banda de acesso restrito (Gualdino, 2011). A terceira geração do BeiDou, iniciada em 2015, busca o estabelecimento de uma constelação com cobertura global.

Informações atualizadas sobre a estrutura dos sinais dos diversos sistemas GNSS podem ser encontradas em Gowdayyanadoddi et al. (2016).

\section{Diluição de Precisão}

A forma em que os satélites estão dispostos geometricamente afeta diretamente a qualidade do posicionamento. O DOP (Dilution of Precision), calculado com base na posição da estação de interesse e dos satélites visíveis a partir dela, expressa a propagação dos erros de observação nos parâmetros da estação (coordenadas e tempo) em função da geometria da constelação. A Equação 1 expressa em linhas gerais esta relação. 
Quando os satélites se encontram muito próximos entre si, obtém-se um valor de DOP alto decorrente de uma geometria fraca. Portanto, para obter resultados com melhor precisão é crucial ter valores baixos de DOP, o que ocorre quanto mais afastados entre si estiverem os satélites visíveis (Gualdino, 2011).

$\mathrm{O}$ volume da figura geométrica formada pelos vetores unitários que unem os satélites e o receptor é inversamente proporcional ao DOP, ou seja, quanto mais dispersos estiverem os satélites visíveis, maior será o volume e, assim, menor será a propagação dos erros de observação, ocasionada pela geometria dos satélites, nos resultados do posicionamento (Monico, 2000). Para examinar a diluição de precisão em parâmetros de interesse específicos, o DOP pode assumir as seguintes formas: GDOP (Geometric DOP) - representa a contribuição da geometria na qualidade da posição tridimensional e do tempo; PDOP (Position DOP) - contribuição da geometria na qualidade da posição tridimensional; HDOP (Horizontal DOP) contribuição na qualidade da posição horizontal (latitude e longitude); VDOP (Vertical DOP) - contribuição na qualidade da posição vertical (altitude geométrica); TDOP (Time DOP) - contribuição na qualidade da determinação do tempo (ou seja, do erro do relógio do receptor).

\section{Metodologia}

A metodologia deste trabalho baseia-se na elaboração de gráficos DOP, através do uso do serviço GNSS Planning Online (Trimble, 2015), e no processamento das observações GNSS no serviço IBGE-PPP (IBGE, 2015) e no software RTKLIB (Takasu, 2013), conforme os esquemas apresentados nas Figuras 1 e 2.

\section{Dados utilizados}

Os dados utilizados nesta pesquisa são de estações GNSS localizadas no estado de São Paulo. As estações são identificadas pelas siglas SPAR, SPC1, SPTU e CHPG, situadas nos municípios de Araçatuba, Campinas, Tupã, e Cachoeira Paulista, respectivamente (Figura 3).

Os dados GNSS foram coletados nestas estações com receptores TRIMBLE NETR9. As três primeiras pertencem à RBMC (Rede Brasileira de Monitoramento dos Sistemas GNSS) (FORTES et al., 1998), enquanto a quarta pertence ao experimento MGEX (Multi-GNSS Experiment) do Serviço Internacional GNSS (IGS) (IGS-MGEX, 2015). Cabe destacar que as quatro estações contribuem para a densificação da rede global IGS, garantindo uma melhor precisão dos produtos IGS sobre o território brasileiro.

A Tabela 1 apresenta as coordenadas de cada estação, assim como a agência responsável e os sistemas capazes de rastrear. As observações de SPAR, SPTU e 

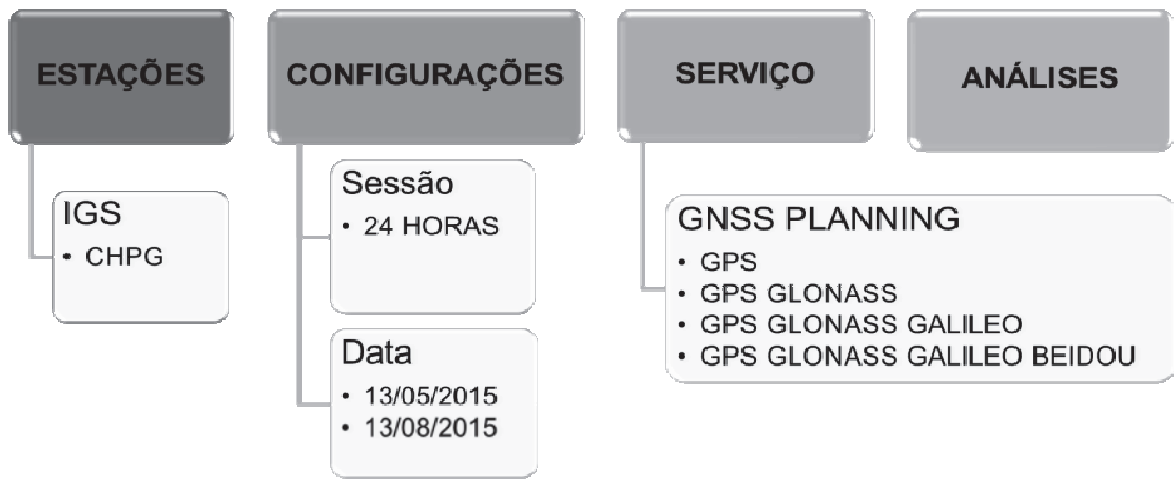

Figura 1. Esquema de elaboração de gráficos DOP.

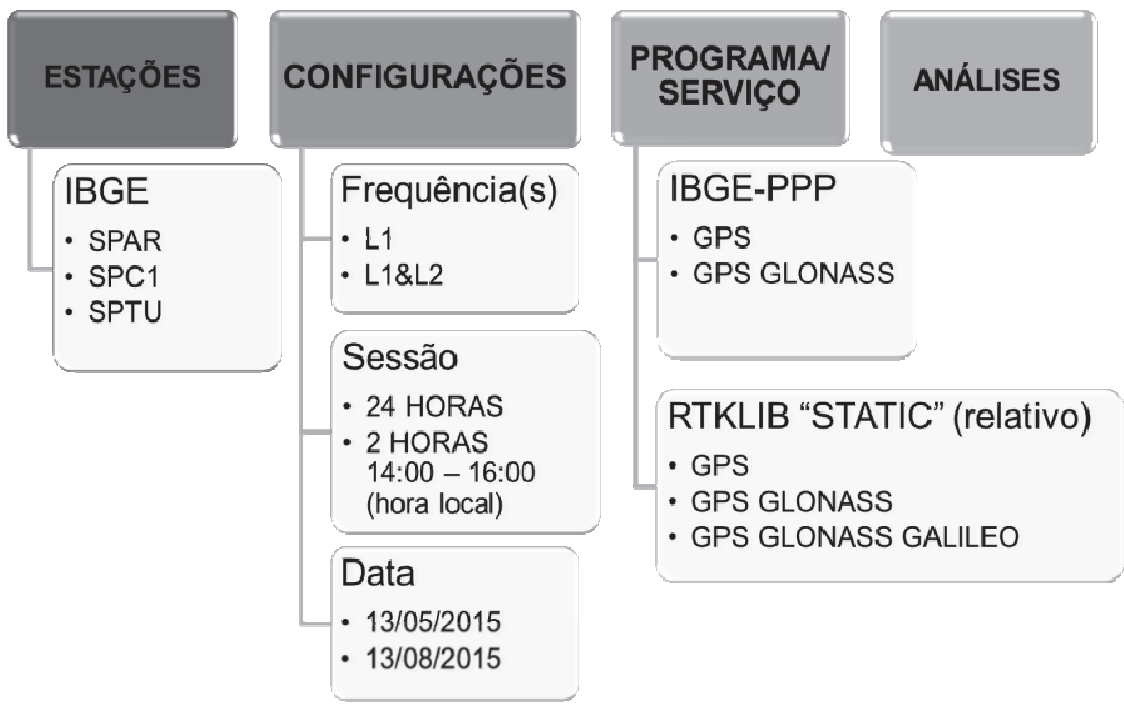

Figura 2. Esquema de processamentos de posicionamento preciso por ponto (IBGE-PPP) e relativo (RTKLIB). 


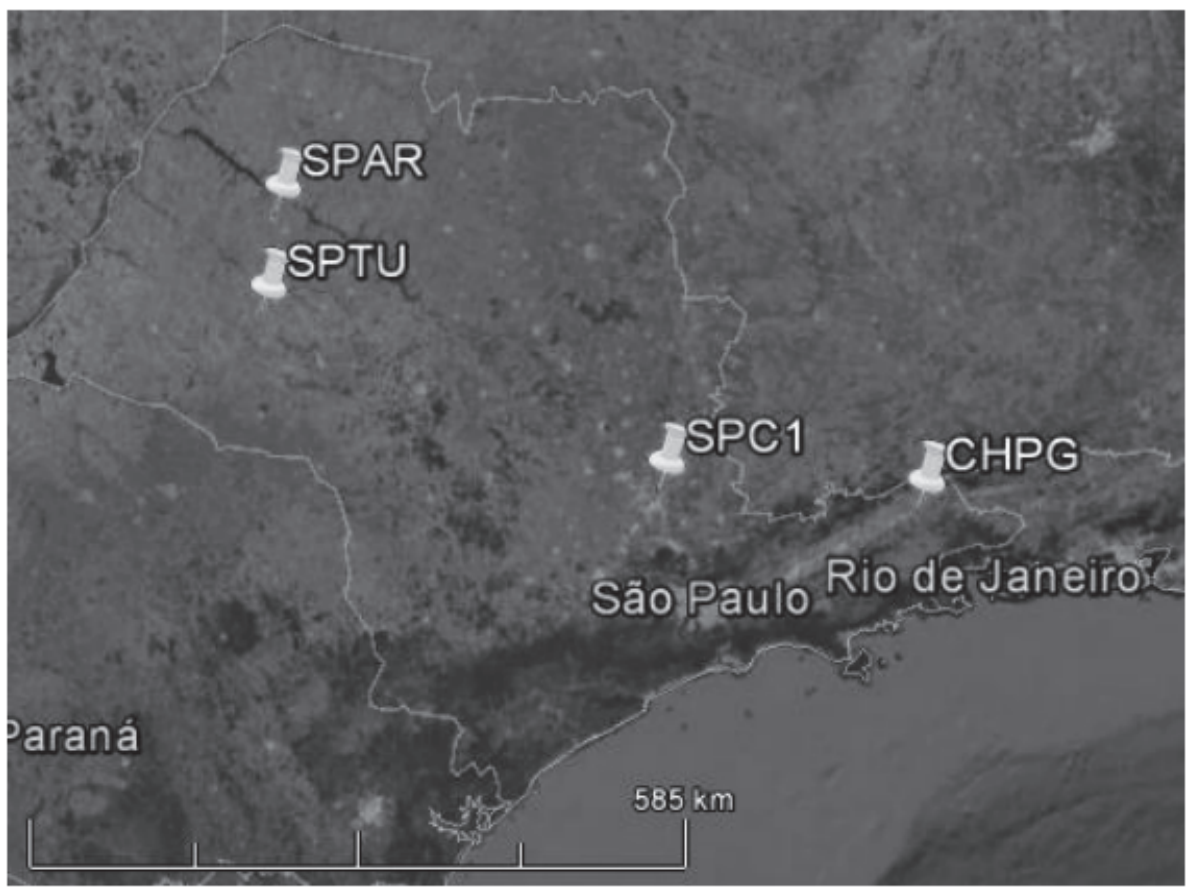

Figura 3. Localização das estações.

Fonte: Google Earth, 2015.

Tabela 1

Informações gerais de cada estação

\begin{tabular}{l|llll}
\hline \multicolumn{1}{c}{ Estação } & \multicolumn{1}{c}{ SPTU } & \multicolumn{1}{c}{ SPAR } & \multicolumn{1}{c}{ SPC1 } & \multicolumn{1}{c}{ CHPG } \\
\hline \multirow{2}{*}{ Cidade } & Tupã - SP & Araçatuba - SP & Campinas - SP & $\begin{array}{l}\text { Cachoeira } \\
\text { Paulista - SP }\end{array}$ \\
Agência & IBGE & IBGE & IBGE & CNES \\
& GPS + GLO + & GPS + GLO + & GPS + GLO + & GPS + GLO + \\
Sistemas & GAL & GAL & GAL & SBAS \\
& 508,783 & 410,351 & 622,980 & 410,351 \\
Altitude (m) & $-21^{\circ} 55^{\prime} 44,82^{\prime \prime}$ & $-21^{\circ} 11^{\prime} 04,80^{\prime \prime}$ & $-22^{\circ} 48^{\prime} 58,63^{\prime \prime}$ & $-22^{\circ} 400^{\prime} 55,90^{\prime \prime}$ \\
Latitude & $-50^{\circ} 29^{\prime} 32,53^{\prime}$, & $-50^{\circ} 26^{\prime} 23,24^{\prime \prime}$ & $-47^{\circ} 03^{\prime} 45,70^{\prime \prime}$ & $-45^{\circ} 00^{\prime} 07,99^{\prime \prime}$ \\
Longitude & & &
\end{tabular}

Fonte: Adaptado dos descritivos das estações do IBGE e IGS. 
SPC1 são disponibilizadas pelo IBGE, em http:/www.ibge.gov.br/, no link Download, na área de Geociências, acessando a pasta RBMC. As observações de CHPG e efemérides precisas dos satélites GPS, GLONASS, Galileo e BeiDou foram descarregadas a partir da iniciativa MGEX do IGS (IGS-MGEX, 2015). O MGEX objetiva realizar experimentos multi-GNSS, disponibilizando dados dos sistemas que ainda estão em desenvolvimento, tais como o Galileo e o BeiDou.

Os dados utilizados estão em formato RINEX 2.11, com 24 horas de observação e taxa de coleta de 15 segundos, para as estações pertencentes ao IBGE, e formato RINEX 3.02, com taxa de coleta de 30 segundos, para a estação CHPG.

Foram selecionados dados coletados nestas estações nos dias 13/05/2015 e $13 / 08 / 2015$. As datas foram determinadas em função do alto e baixo conteúdo total de elétrons (TEC, na sigla em inglês) na atmosfera nestes períodos. O primeiro, menos de dois meses após o equinócio de março, atingindo um valor vertical máximo de 75 TEC units (TECU), e o segundo, no inverno do hemisfério sul, atingindo um máximo de 25 TECU (1 TECU corresponde a um erro de 0,163 m na distância receptor-satélite medida com L1 devido à refração ionosférica Klobuchar, 1996). Cabe registrar que o período de coleta de dados foi relativamente próximo ao último pico de atividade solar, ocorrido no entorno de 2013, do último ciclo solar de 11 anos (Fortes et al., 2015). Alterações no campo magnético da Terra provocadas pela atividade solar são monitorados por observatórios magnéticos terrestres, utilizando índices geomagnéticos para quantificarem tais variações. Dois desses índices geomagnéticos são: Kp (Planetarische Kennziffer) e Ap (correspondente linear do Kp). O índice Kp reflete a instabilidade na ionosfera e revela a ocorrência ou não de tempestades geomagnéticas. Seus valores variam em uma escala crescente de 0 a 9, caracterizando a ocorrência de tempestades geomagnéticas quando igual ou superior a 5. O índice Ap é o correspondente linear do índice Kp, cujos valores variam de 0 a 400, e expressa o intervalo de perturbação do campo geomagnético. Nas datas 13/05/2015 e 13/08/2015 verificou-se que o índice Kp variou de 4 a 6 e de 1 a 4, e o índice Ap variou de 32 a 67 e de 4 a 32, respectivamente, confirmando a ocorrência de maior instabilidade ionosférica no dia 13 de maio de 2015 .

\section{Processamentos}

Os processamentos foram feitos nos programas/serviços descritos, para os dias 13/05/2015 e 13/08/2015. No caso do IBGE-PPP e RTKLIB, foram utilizados dados coletados em sessões de duas e 24 horas de duração, utilizando uma (L1) e duas (L1 e L2) frequências. As sessões de duas horas foram selecionadas no entorno das $14 \mathrm{~h}$ do horário local, período do dia de maior incidência da refração ionosférica (Fortes, 2002). 


\section{GNSS Planning Online}

O GNSS Planning Online é um serviço oferecido pela Trimble, que utiliza dados do almanaque dos satélites, no qual permite ao usuário ter acesso a diversas informações GNSS, o nível de atividade ionosférica e o DOP na época de interesse. $\mathrm{Na}$ página inicial, o usuário configura as máscaras de elevação dos satélites, latitude, longitude e altitude geométrica do lugar, data, hora inicial e período de interesse. O usuário também pode escolher diversas opções de constelação, entre elas, GPS, GLONASS, Galileo e BeiDou. O serviço permite determinar o melhor momento para observar os satélites em uma determinada estação.

Este serviço foi utilizado para gerar gráficos de DOP e do número de satélites disponíveis no momento do posicionamento, com máscara de elevação de dez graus, para a estação CHPG. Os gráficos de DOP foram gerados considerando as combinações dos sistemas GPS, GPS + GLONASS, GPS + GLONASS + Galileo e GPS + GLONASS + Galileo + Beidou.

\section{$I B G E-P P P$}

O IBGE disponibiliza um serviço online gratuito, denominado IBGE-PPP, para o pós-processamento de dados GNSS utilizando a técnica de Posicionamento Preciso por Ponto (PPP). Esse serviço permite aos usuários com receptores GPS ou GPS/GLONASS obterem coordenadas referidas ao Sistema Geodésico Brasileiro (SIRGAS2000), época 2000,4. O IBGE-PPP processa dados GPS e GLONASS, em formato RINEX, que foram coletados por receptores de uma ou duas frequências, no modo estático ou cinemático. Em cada processamento, o IBGE-PPP aplica a correção do centro de fase das antenas dos satélites e receptores, parâmetros de transformação ITRF/SIRGAS2000, modelo de carga oceânica, modelo sulamericano de velocidades VEMOS2009 (Drewes e Heidbach, 2012) e a versão oficial do Modelo de Ondulação Geoidal do Brasil - MAPGEO.

Utilizou-se o IBGE-PPP para processar dados GPS e GPS + GLONASS coletados nas estações SPTU, SPAR e SPC1. Para as sessões de duas horas de duração e/ou apenas uma frequência, o arquivo diário das observações RINEX correspondente foi editado, excluindo-se as observações indesejadas.

\section{RTKLIB}

O RTKLIB é um software livre que processa dados coletados por receptores GNSS, tais como os que rastreiam os sistemas GPS, GLONASS, Galileo, BeiDou, QZSS e SBAS, para o posicionamento absoluto ou relativo, cinemático ou estático, em pósprocessamento ou em tempo real.

O aplicativo RTKPOST, do RTKLIB, foi utilizado para executar o processamento relativo dos dados GPS, GPS + GLONASS e GPS + GLONASS + 
Galileo, coletados nas estações SPTU, SPAR e SPC1, utilizando-se efemérides precisas. Para este estudo, o software foi configurado com as seguintes especificações: modo de posicionamento "Static"; frequências: "L1" ou "L1 + L2" (Backward); máscara de elevação de $10^{\circ}$; correção ionosférica "off" ou "iono-free"; correção troposférica "Estimate ZTD"; e solução de ambiguidade "Continuous".

\section{Resultados e discussão}

Nesta seção, são apresentados os resultados e análises com a finalidade de verificar os impactos causados pelo uso dos diferentes sistemas no posicionamento por satélites.

\section{DOP}

As Figuras 4 a 11 apresentam os gráficos dos diversos tipos de DOP (da esquerda para a direita da legenda: GDOP, TDOP, PDOP, VDOP e HDOP) gerados pelo GNSS Planning Online para a estação CHPG para os dias 13 de maio e 13 de agosto de 2015. Deve-se atentar que a escala do eixo vertical varia ao longo das figuras.

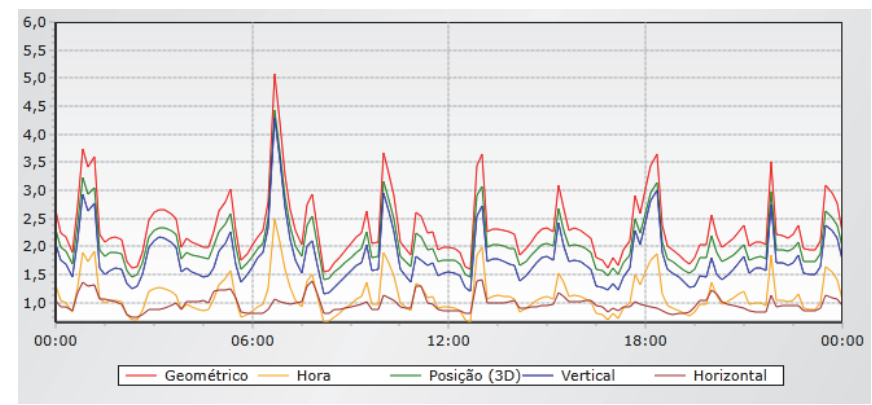

Figura 4. DOP na estação CHPG dos satélites GPS em 13 de maio de 2015.

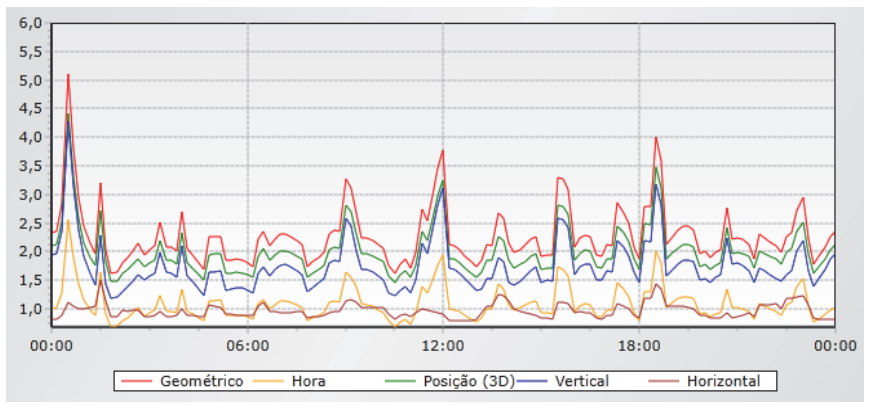

Figura 5. DOP na estação CHPG dos satélites GPS em 13 de agosto de 2015. 
42 Samara Chamorro Navarro Ferreira et al. $\quad$ Análise preliminar dos impactos do uso de mais...

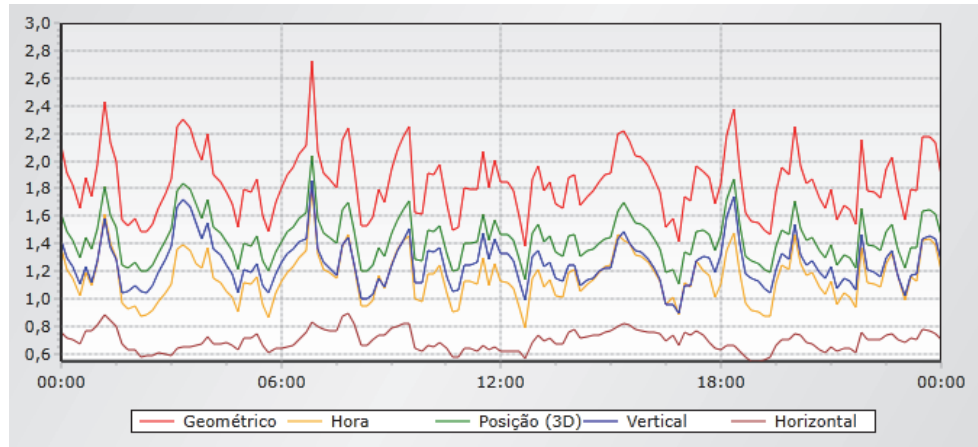

Figura 6. DOP na estação CHPG dos satélites GPS e GLONASS em 13 de maio de 2015.

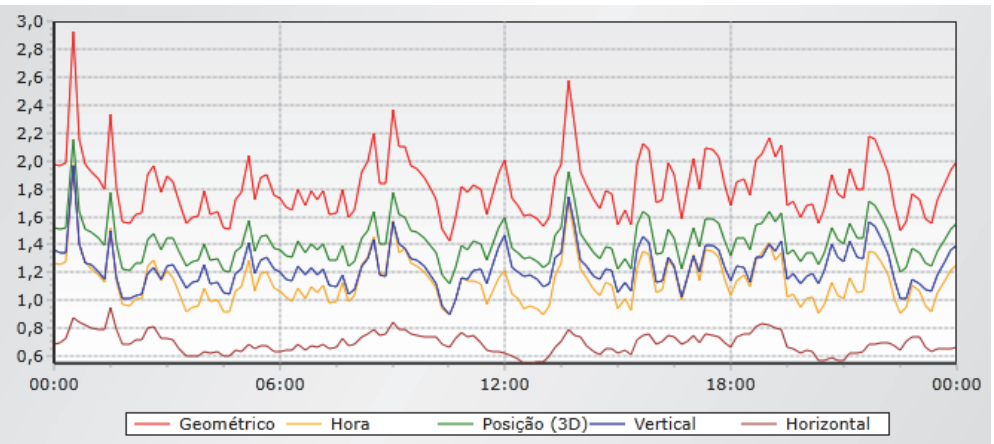

Figura 7. DOP na estação CHPG dos satélites GPS e GLONASS em 13 de agosto de 2015.

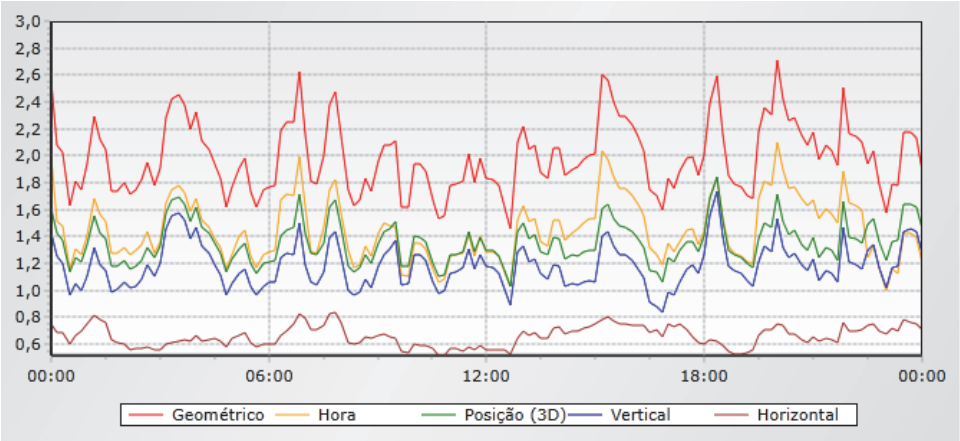

Figura 8. DOP na estação CHPG dos satélites GPS, GLONASS e Galileo em 13 de maio de 2015 . 


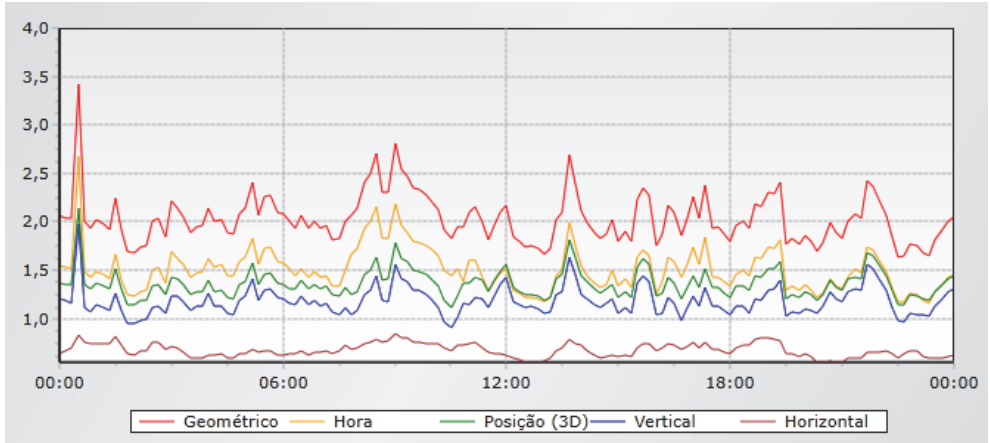

Figura 9. DOP na estação CHPG dos satélites GPS, GLONASS e Galileo em 13 de agosto de 2015.

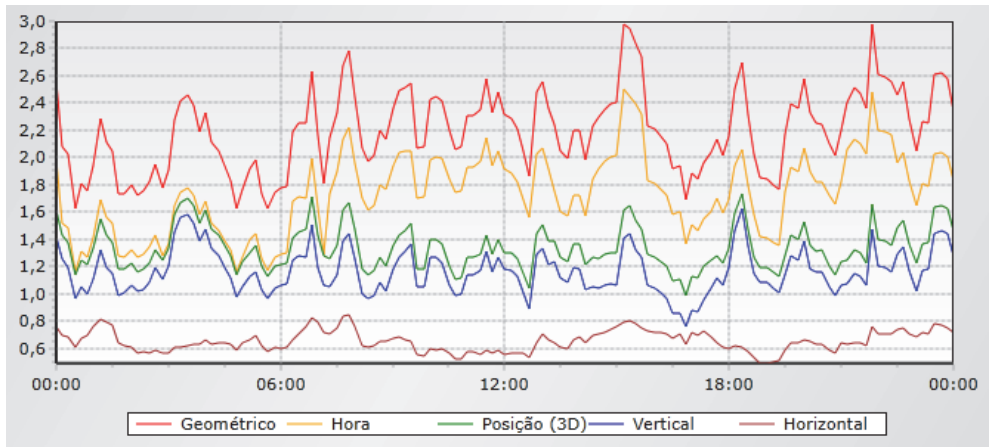

Figura 10. DOP na estação CHPG dos satélites GPS, GLONASS, Galileo e BeiDou em 13 de maio de 2015.

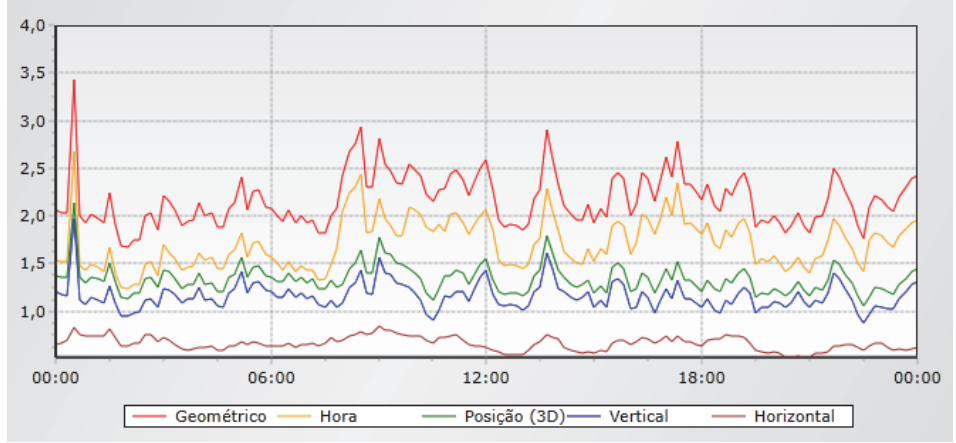

Figura 11. DOP na estação CHPG dos satélites GPS, GLONASS, Galileo e BeiDou em 13 de agosto de 2015. 
O número de satélites influencia diretamente os valores de DOP. Em geral, quanto mais satélites, menor é o DOP e, consequentemente, melhor a geometria. Os gráficos das Figuras 12 e 13 mostram o número de satélites visíveis na estação CHPG nos dois dias em pauta para cada sistema.

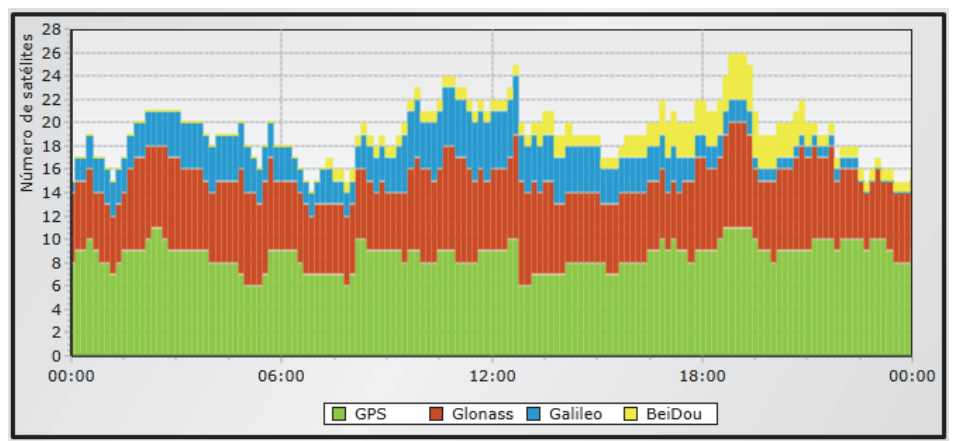

Figura 12. Quantidade de satélites visíveis na estação CHPG no dia 13 de maio de 2015.

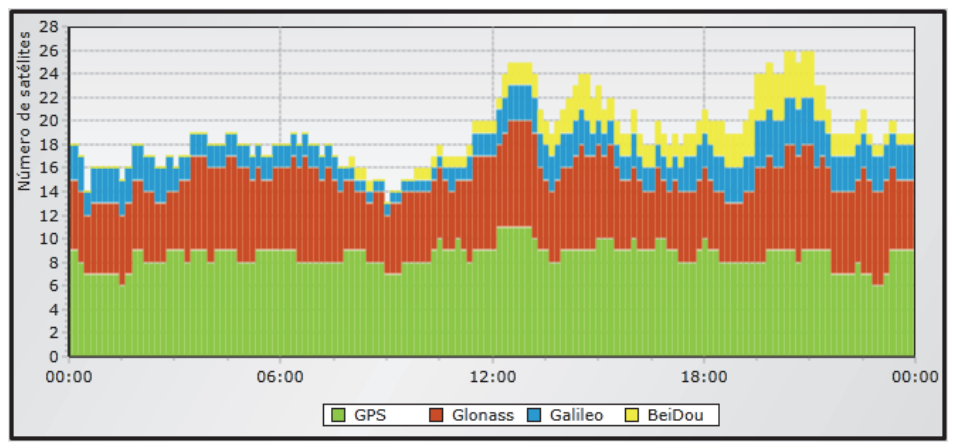

Figura 13. Quantidade de satélites visíveis na estação CHPG no dia 13 de agosto de 2015.

Os valores de PDOP foram utilizados para a análise dos impactos de uso de mais de um sistema feito a seguir.

Observa-se a passagem, em média, de nove satélites GPS durante o intervalo de 24 horas nos dias 13 de maio de 2015 e 13 de agosto de 2015, com um PDOP médio igual a 2. Neste mesmo período, verificam-se aproximadamente seis satélites GLONASS disponíveis, com variação de três satélites para mais ou para menos. O valor do PDOP médio da constelação GPS + GLONASS passa a ser de 1,5, uma melhoria de $25 \%$ quando comparado com o obtido apenas com o GPS.

Até quatro satélites Galileo estiveram visíveis nestes dois dias, contribuindo para um PDOP médio de 1,4, representando uma melhoria de 30\% em relação ao obtido com o GPS e de 7\% em relação ao GPS + GLONASS. 
Em função do estágio do desenvolvimento do sistema BeiDou, observa-se pouca disponibilidade destes satélites na estação CHPG nestes dois dias, com praticamente mais de seis horas em cada dia sem nenhum satélite visível. Foram contabilizados no máximo quatro satélites vistos simultaneamente, durante um curto período de tempo, o que não contribuiu para uma melhoria do PDOP médio do caso anterior.

\section{Discrepância planimétrica e altimétrica}

A qualidade dos posicionamentos foi avaliada através do viés (discrepância), calculado pela diferença entre as coordenadas oriundas dos processamentos no IBGE-PPP ou RTKLIB (posicionamento relativo), e as coordenadas de referência publicadas pelo IBGE. As diferenças planimétricas (DH) e altimétricas (DV), estas últimas em valor absoluto, são apresentadas nas Tabelas 2 a 6 , sendo:

$$
\begin{array}{ll}
24 \mathrm{~h} & - \text { Sessão de rastreio com } 24 \text { horas } \\
2 \mathrm{~h} & - \text { Sessão de rastreio com } 2 \text { horas } \\
\text { L1L2 } & \text { - Rastreio das frequências L1 e L2 } \\
\text { L1 } & \text { - Rastreio da frequência L1 } \\
13-05 & \text { - Data de coleta dos dados (13 de maio de 2015) } \\
13-08 & \text { - Data de coleta dos dados (13 de agosto de 2015) }
\end{array}
$$

Foram utilizadas as estações SPTU, SPAR e SPC1 nesta etapa dos processamentos. A estação CHPG, a única que rastreou dados do BeiDou, apresentou problemas no processamento com o RTKLIB devido a uma incompatibilidade do programa com o formato RINEX 3.02 utilizado no arquivo de dados correspondente.

Os resultados para o caso GPS + GLONASS + Galileo não foram incluídos nas tabelas por apresentarem valores idênticos àqueles obtidos no caso GPS0 + GLONASS. Uma investigação posterior indicou que as poucas observações dos satélites Galileo foram rejeitadas no processamento com o RTKLIB (O IBGE-PPP ainda não processa dados do sistema europeu).

Nos processamentos do IBGE-PPP, verificou-se a melhor qualidade (centimétrica) das soluções de dupla frequência, seja com $24 \mathrm{~h}$ ou $2 \mathrm{~h}$ de dados, como esperado. Neste caso, a inclusão do sistema GLONASS melhorou cerca de $8 \%$ a qualidade do posicionamento. Os arquivos de dados de SPC1 para 13 de maio de 2015 não continham observações em L2, portanto não foi possível gerar soluções de dupla frequência. 
Posicionamento Preciso por Ponto (IBGE PPP: Tabela 2, 3 e 4)

Tabela 2

Discrepância planimétrica e altimétrica do posicionamento preciso por ponto pós-processado no serviço IBGE-PPP da estação SPTU

\begin{tabular}{lcccc}
\hline \multirow{2}{*}{ PPP } & \multicolumn{4}{c}{$S P T U$} \\
\cline { 2 - 5 } & \multicolumn{3}{c}{$G P S$} & GPS GLONASS \\
\cline { 2 - 5 } & $D H(m)$ & $D V(m)$ & $D H(m)$ & $D V(m)$ \\
\hline 24h L1L2 13-05 & 0,024 & 0,033 & 0,022 & 0,013 \\
24h L1L2 13-08 & 0,022 & 0,023 & 0,022 & 0,023 \\
2h L1L2 13-05 & 0,033 & 0,043 & 0,028 & 0,023 \\
2h L1L2 13-08 & 0,04 & 0,013 & 0,036 & 0,023 \\
24h L1 13-05 & 1,764 & 0,363 & 1,739 & 0,323 \\
24h L1 13-08 & 0,086 & 1,163 & 0,083 & 1,223 \\
2h L1 13-05 & 2,782 & 0,463 & 2,814 & 0,363 \\
2h L1 13-08 & 0,294 & 1,083 & 0,352 & 1,233 \\
\hline
\end{tabular}

Tabela 3

Discrepância planimétrica e altimétrica do posicionamento preciso por ponto pós-processado no serviço IBGE-PPP da estação SPAR

\begin{tabular}{|c|c|c|c|c|}
\hline \multirow{3}{*}{$P P P$} & \multicolumn{4}{|c|}{$S P A R$} \\
\hline & \multicolumn{2}{|c|}{ GPS } & \multicolumn{2}{|c|}{ GPS GLONASS } \\
\hline & $D H(m)$ & $D V(m)$ & $D H(m)$ & $D V(m)$ \\
\hline 24h L1L2 13-05 & 0,022 & 0,031 & 0,023 & 0,031 \\
\hline 24h L1L2 13-08 & 0,019 & 0,031 & 0,019 & 0,031 \\
\hline 2h L1L2 13-05 & 0,036 & 0,051 & 0,028 & 0,051 \\
\hline 2h L1L2 13-08 & 0,03 & 0,021 & 0,028 & 0,021 \\
\hline 24h L1 13-05 & 1,707 & 0,881 & 1,671 & 0,871 \\
\hline 24h L1 13-08 & 0,086 & 1,261 & 0,875 & 1,331 \\
\hline 2h L1 13-05 & 2,477 & 1,441 & 2,634 & 1,341 \\
\hline 2h L1 13-08 & 0,202 & 1,331 & 0,318 & 1,321 \\
\hline
\end{tabular}


Tabela 4

Discrepância planimétrica e altimétrica do posicionamento preciso por ponto pós-processado no serviço IBGE-PPP da estação SPC1

\begin{tabular}{lcccc}
\hline \multirow{2}{*}{ PPP } & \multicolumn{4}{c}{ SPC1 } \\
\cline { 2 - 5 } & \multicolumn{3}{c}{$G P S$} & GPS GLONASS \\
\cline { 2 - 5 } & $D H(m)$ & $D V(m)$ & $D H(m)$ & $D V(m)$ \\
\hline 24h L1L2 13-05 & 0,022 & 0,012 & $*$ & $*$ \\
24h L1L2 13-08 & 0,022 & 0,011 & 0,022 & 0,012 \\
2h L1L2 13-05 & 0,025 & 0,004 & $*$ & $*$ \\
2h L1L2 13-08 & 0,031 & 0,002 & 0,031 & 0,000 \\
24h L1 13-05 & 1,916 & 0,479 & 1,858 & 0,477 \\
24h L1 13-08 & 0,120 & 1,138 & 0,122 & 1,192 \\
2h L1 13-05 & 3,024 & 0,828 & 2,994 & 1,111 \\
2h L1 13-08 & 0,184 & 1,392 & 0,249 & 1,287 \\
\hline
\end{tabular}

Considerando receptores de apenas uma frequência, o uso do GLONASS não apresentou mudanças significativas na qualidade do posicionamento; pois quando comparado com a magnitude dos erros, tendo em vista estes serem dominados pela refração ionosférica incidente sobre as observações, a inclusão do GLONASS possui menor influencia. Cabe destacar que estes erros atingiram valores maiores para o dia 13 de maio de 2015, por ser mais afetado pela ionosfera.

\section{Posicionamento Relativo (Tabela 5 e 6)}

O RTKLIB foi utilizado para processar dados GPS e GPS + GLONASS no modo relativo. A estação SPTU foi usada como estação de referência, com linhas de base de 82.607,306 m para SPAR e 366.624,312 m para a SPC1.

Analogamente ao observado nos processamentos com o IBGE-PPP, verificou-se a melhor qualidade (centimétrica) das soluções de dupla frequência, seja com $24 \mathrm{~h}$ ou $2 \mathrm{~h}$ de dados, como era de se esperar. Assim como no posicionamento preciso por ponto, a inclusão do sistema GLONASS melhorou cerca de $8 \%$ a qualidade do posicionamento. 
Tabela 5

Discrepância planimétrica e altimétrica do posicionamento relativo pós-processado no RTKLIB da estação SPAR

\begin{tabular}{lcccc}
\hline \multirow{2}{*}{\multicolumn{1}{c}{$P R$}} & \multicolumn{4}{c}{ GPTU $>$ SPAR } \\
\cline { 2 - 5 } & $D H(m)$ & $D V(m)$ & $D H(m)$ & $D V(m)$ \\
\hline 24h L1L2 13-05 & 0,011 & 0,052 & 0,012 & 0,056 \\
24h L1L2 13-08 & 0,017 & 0,055 & 0,015 & 0,056 \\
2h L1L2 13-05 & 0,021 & 0,062 & 0,018 & 0,065 \\
2h L1L2 13-08 & 0,034 & 0,041 & 0,029 & 0,043 \\
24h L1 13-05 & 0,413 & 0,331 & 0,333 & 0,405 \\
24h L1 13-08 & 0,027 & 0,023 & 0,018 & 0,021 \\
2h L1 13-05 & 0,91 & 0,797 & 0,878 & 0,816 \\
2h L1 13-08 & 0,18 & 0,086 & 0,159 & 0,053 \\
\hline
\end{tabular}

Tabela 6

Discrepância planimétrica e altimétrica do posicionamento relativo pós-processado no RTKLIB da estação SPC1

\begin{tabular}{|c|c|c|c|c|}
\hline \multirow{3}{*}{$P R$} & \multicolumn{4}{|c|}{$S P T U>>S P C 1$} \\
\hline & \multicolumn{2}{|c|}{$G P S$} & \multicolumn{2}{|c|}{ GPS GLONASS } \\
\hline & $D H(m)$ & $D V(m)$ & $D H(m)$ & $D V(m)$ \\
\hline 24h L1L2 13-05 & 0,024 & 0,024 & $*$ & * \\
\hline 24h L1L2 13-08 & 0,019 & 0,018 & 0,017 & 0,019 \\
\hline 2h L1L2 13-05 & 0,015 & 0,013 & $*$ & $*$ \\
\hline 2h L1L2 13-08 & 0,018 & 0,016 & 0,011 & 0,002 \\
\hline 24h L1 13-05 & 0,705 & 1,134 & 0,348 & 0,715 \\
\hline 24h L1 13-08 & 0,159 & 0,041 & 0,139 & 0,035 \\
\hline 2h L1 13-05 & 1,436 & 4,498 & 1,224 & 2,19 \\
\hline 2h L1 13-08 & 0,33 & 0,573 & 0,315 & 0,601 \\
\hline
\end{tabular}

No posicionamento com apenas uma frequência, cabe registrar que os erros, tanto com dados GPS quanto com os GPS + GLONASS, se reduzem em relação àqueles obtidos na solução IBGE-PPP na maioria dos casos, especialmente na linha de base mais curta (SPTU>>SPAR). Esta redução pode ser atribuída ao cancelamento de parte dos erros ionosféricos no processamento relativo. Entretanto, no caso da linha de base mais longa (SPTU $>>S P C 1$ ), estes erros ainda atingiram valores métricos no dia mais afetado pela ionosfera (13 de maio de 2015), tendo em vista a menor eficácia da redução dos erros ionosféricos. Nestas soluções de uma frequência, a inclusão do sistema russo foi vantajosa, a qualidade do posicionamento melhorou aproximadamente $17 \%$. 


\section{Conclusões}

A partir da análise dos valores de DOP, verificou-se que o acréscimo de medidas GLONASS juntamente com medidas GPS no posicionamento indica uma melhoria da qualidade do mesmo, uma vez que o PDOP médio variou de 2 para 1,5 (redução de $25 \%$ ). Os posicionamentos por ponto preciso e relativo com as frequências L1 e L2 apresentaram melhora de aproximadamente $8 \%$ quando comparadas as soluções entre GPS e GPS + GLONASS. Nestes casos, pode-se atribuir à alta acurácia das soluções GPS o fato da adição do GLONASS ter tido pouca influência nos resultados. Ao analisar apenas uma frequência, a melhoria da qualidade das coordenadas também foi observada, sendo o impacto mais significativo no posicionamento relativo, aproximadamente $17 \%$, tendo em vista que os erros das coordenadas no caso do PPP são contaminados pela refração ionosférica. Neste último caso, o serviço IBGE-PPP utiliza a melhor correção ionosférica disponível no momento do processamento, sendo dada preferência aos arquivos disponibilizados pelo IGS (final e rápido), e depois pelas correções disponíveis nos arquivos de efemérides transmitidas (IBGE, 2013). Por conta da alta variabilidade temporal e espacial da ionosfera, principalmente em regiões equatoriais como a do Brasil, a eficácia destas correções é significativamente inferior àquela obtida com o uso de observações de dupla frequência, onde praticamente $100 \%$ do erro é eliminado.

$\mathrm{O}$ acréscimo do sistema europeu às constelações GPS e GLONASS contribuiu para uma redução no valor médio de PDOP em 7\% (1,4 versus 1,5). Sendo assim, avalia-se que a mobilização, neste momento, de receptores capazes de rastrear GPS, GLONASS e Galileo no posicionamento preciso depende da aplicação específica, onde o ganho em acurácia deve ser confrontado com os custos mais elevados dos receptores deste tipo.

Em função do ainda reduzido número de satélites $\mathrm{MEO}$ na constelação do sistema BeiDou, não houve redução no PDOP médio em relação ao obtido com o uso das constelações GPS + GLONASS + Galileo.

As conclusões deste trabalho estão diretamente correlacionadas ao estágio de desenvolvimento dos sistemas Galileo e BeiDou à época da coleta de dados, em maio e agosto de 2015. Entende-se que, na medida em que novos satélites destes sistemas forem lançados, os impactos observados nas atividades de posicionamento podem ser diferentes.

Os resultados apresentados foram obtidos em condições de horizonte livre de obstruções nas estações utilizadas, todas de operação contínua. Evidentemente, os impactos do uso de mais de um sistema GNSS devem se mostrar mais representativos em situações com ocorrência de obstruções, tais como aquelas existentes em cânions urbanos. 


\section{Referências}

Drewes, H. and O. Heidbach (2012). The 2009 Horizontal Velocity Field for South America and the Caribbean, in Kenyon S.; M.C. Pacino and U. Marti (eds.), "Geodesy for Planet Earth", IAG Symposia, 136: 657-664.

Figueirêdo, D.C. (2005). Curso Básico de GPS. Disponível em $<$ http://www.leb.esalq.usp.br/disciplinas/Topo/leb450/Angulo/Curso_GPS.pdf>

Fortes, L.P.S. (2002). Optimising the Use of GPS Multi-Reference Stations for Kinematic Positioning, PhD Thesis, UCGE Report Number 20158, The University of Calgary.

Fortes, L.P.S.; Lin, T. y Lachapelle, G. (2015). Effects of the 2012-2013 solar maximum on GNSS signals in Brazil, GPS Solutions, 19:309-319.

Fortes, L.P.S.; Luz, R.T.; Pereira, K.D.; Costa, S.M.A. and Blitzkow, D. (1998). The Brazilian network for continuous monitoring of GPS (RBMC): operation and products, in Brunner, F.K. (ed.), Advances in positioning and reference frames, International Association of Geodesy Symposia, Springer, vol. 118, pp. 73-78.

Gowdayyanadoddi, N.; Broumandan, A. and Lachapelle, G. (2016). Recording of GNSS Digital Data. White Paper, ESA GSAC-18 Meeting, Paris.

Gualdino, C.M. (2011). Características e desempenho de sistemas de posicionamento global. Disponível em: <https://fenix.tecnico.ulisboa.pt/ downloadFile/395142775308/Tese_Claudio_Gualdino.pdf $>$. Acesso em: 13 de Agosto de 2015.

IBGE. Posicionamento por Ponto Preciso. Disponível em <http://www.ppp. ibge.gov.br/>, Acesso em: 14 de Novembro de 2015.

- (2013). Manual do Usuário Aplicativo Online IBGE-PPP. Disponível em $<$ http://www.ibge.gov.br/home/geociencias/geodesia/ppp/manual_ppp.pdf $>$

IGS. International GNSS Service. Disponível em <http://www.igs.com>. Acesso em 4 de Novembro de 2015.

IGS-MGEX. The Multi-GNSS Experiment and Pilot Project (MGEX). Disponível em http://mgex.igs.org/. Acesso em 4 de novembro de 2015.

Klobuchar, J.A. (1996). Ionopsheric Effects on GPS, in Parkinson, B.W. and Spilker Jr., J.J. (eds.), Global Positioning System: Theory and Applications. Volume I, American Institute of Aeronautics and Astronautics, Inc.Kp index. Disponível em <http://wdc.kugi.kyoto-u.ac.jp/kp/>. Acesso em 11 de Setembro de 2016.

Monico, J.F.G. (2008). Posicionamento pelo GNSS - Descrição, fundamentos e aplicações, Editora UNESP, 2a. edição.

NOAA. Geomagnetic $\mathrm{kp}$ and ap Indices. Disponível em <http://www.ngdc. noaa.gov/stp/GEOMAG/kp_ap.html>. Acesso em 11 de Setembro de 2016. 
Parkinson, B. (1996). Introduction and Heritage of NAVSTAR, the Global Positioning System, in Parkinson, B.W. and Spilker Jr., J.J. (eds.), Global Positioning System: Theory and Applications, Volume I. American Institute of Aeronautics and Astronautics, Inc.

Takasu, T. (2013) RTKLIB ver. 2.4.2 Manual. Disponível em <http://www. rtklib.com/prog/manual_2.4.2.pdf $>$, acesso em: 6 de Junho de 2015. 